sexual activity when calculating Chlamydia incidence and screening rates, particularly for adolescents. Using data from a provincial adolescent health survey we assessed the impact of adjusting for sexual activity on population-based Chlamydia incidence and screening rates among adolescents in British Columbia (BC), Canada.

Methods We estimated the proportion of adolescent males (15-18 years) and females (14-18 years) who had ever had sexual intercourse (i.e., sexually active) using data from a cluster-stratified survey of public school students (Grades 7-12) completed by 30,000 BC students in 2003 and 2008. Using provincial Chlamydia surveillance and testing data we compared adolescent Chlamydia screening and incidence rates in BC by age and gender, using total and sexually active populations as denominators.

Results During these time periods, an estimated $32 \%$ and $33 \%$ of males $15-18$ years and $28 \%$ and $31 \%$ of females $14-18$ years were sexually active in 2003 and 2008 respectively. Regardless of denominator used, screening and incidence rates increased with age, and were higher among females compared to males. Sexually active incidence and screening rates were consistently higher with a more pronounced impact at younger ages. For example, in 2008 screening rates among 14 year old females were $26.2 \%$ vs $2.5 \%$ in sexually active and total populations respectively, while the corresponding rates among 18 year old females were $60.2 \%$ vs $28.9 \%$ (2.1 times higher).

Conclusions Using data representing the entire population of $\mathrm{BC}$ adolescents we demonstrated that without adjustment for sexual behaviour, adolescent Chlamydia incidence and screening rates are substantially under-estimated, particularly at younger ages. Adjusting for sexual behaviour using population survey data is essential for accurately monitoring the population impact of prevention and screening programmes among adolescents.

\section{P3.316 ESTIMATING CHLAMYDIA AND GONORRHOEA BURDEN WITHIN THE US ARMY - A REVIEW OF PASSIVE SURVEILLANCE SYSTEMS TO IDENTIFY INCIDENT INFECTIONS}

doi:10.1136/sextrans-2013-051184.0770

'N N Jordan, ${ }^{1,2} \mathrm{~N} \mathrm{~K}$ Leamer, ${ }^{3} \mathrm{G}$ Nowak, ${ }^{4} \mathbf{J}$ C Gaydos. ${ }^{1}$ US Army Institute of Public Health, Aberdeen Proving Grounds-South, MD, United States; ${ }^{2}$ Oak Ridge Institure of Science and Education, Oak Ridge, TN, United States; 'Navy Marine Corps Public Health Center, Norfolk, VA, United States; ${ }^{4}$ Armed Forces Health Surveillance Center, Silver Spring, MD, United States

Background The burden of sexually transmitted infections (STI) within the US military is primarily assessed through review of case reports for notifiable conditions (e.g., Chlamydia, gonorrhoea, and syphilis). The degree to which under-reporting affects burden approximations is unknown. This study was conducted to assess the burden of Chlamydia and gonorrhoea infections and compare case capture across multiple military medical data systems.

Methods Incident infections among Army active duty soldiers from January 2007-December 2011 were identified using three data sources: Health Level 7 laboratory records (HL7), Military Health System Data Repository (MDR) medical records, and case reports from the Disease Reporting System internet (DRSi) and its predecessor, the Reportable Medical Event System (RMES). A thirty day rule was used to define incident cases; i.e., successive records logged within 30 days were excluded.

Results 53,228 incident Chlamydia infections and 11,065 incident gonorrhoea infections were identified over five years; calendar year 2011 incidence rates were 22.0 and 4.2 infections per 1000 personyears, respectively. Chlamydia case capture was $80 \%, 74 \%$, and $35 \%$, respectively for case reports, HL7, and medical records. Gonorrhea case capture was $65 \%, 62 \%$, and $56 \%$, respectively for medical records, case reports and HL7. A notable decrease in laboratory identified infections occurred in 2011, decreasing from highs of $78 \%$ and
$60 \%$ in 2008 to $67 \%$ and $49 \%$ for Chlamydia and gonorrhoea, respectively.

Conclusion The results emphasise the need to utilise multiple databases to obtain more complete estimates of STI burden among active duty Army personnel. While this comprehensive approach provides a better approximation of burden, estimates are likely conservative since many infections go undetected or undocumented. Future analyses should incorporate advanced statistical methods such as capture-recapture methodology to estimate infections not identified through conventional passive surveillance. Furthermore, the decreased case detection through laboratory records is notable, and worthy of investigation.

\section{P3.317 USE OF TABLETS FOR DATA COLLECTION AMONG FEMALE SEX WORKERS: LESSONS LEARNED FROM A BEHAVIOURAL SURVEILLANCE STUDY IN HONDURAS, 2012}

doi:10.1136/sextrans-2013-051184.0771

${ }^{1} \mathbf{N}$ Farach, ${ }^{2 \mathrm{H}}$ Galindo, ${ }^{3} \mathrm{~F}$ Tinajeros, ${ }^{4} \mathrm{M}$ Guardado. ${ }^{1} \mathrm{CDC} / \mathrm{CAR} / \mathrm{DGHA}$, Tegucigalpa, Honduras, ${ }^{2}$ Secretaria de Salud, Tegucigalpa, Honduras, ${ }^{3}$ TEPHINET, Tegucigalpa, Honduras, 4TEPHINET, Guatemala, Guatemala

Background The use of tablets and other hand-held devices is increasingly considered as an option for survey data collection, particularly sensitive personal information among key populations.

Methods In 2012, a behavioural surveillance study was conducted among 1426 female sex workers (FSWs) in Honduras. Sensitive personal information was collected, including sexual practises, condom use, sexual violence, alcohol and drug use, and reporting of STI symptoms. Research instruments were inputted into tablets and piloted with FSWs. During the study, facilitators briefed participants in tablet use while participants inputted their responses, in case they had any issues. Daily occurrences were recorded into a log. We analysed qualitative pilot results reports and discussed the advantages and disadvantages of using tablets for data collection with survey facilitators and investigators during regular monitoring visits.

Results Tablet use eliminated the need for paper questionnaires and data entry, and allowed for early database population as surveys were uploaded to a virtual platform. Facilitator help was repeatedly required by participants and self-administered surveys often took more time to complete, particularly during the first sections when participants were familiarising themselves with the tablet. Issues with web connectivity prevented data submission as scheduled in some sites and two tablets were reported missing in one site (out of a total of 22 tablets in the study).

Conclusions The use of tablets can reduce certain study costs (data input, photocopying) and facilitate data management. Facilitators should be available to assist survey participants as they input their own responses, particularly in settings where participants have had little exposure to technology. A brief introduction to tablet use and practise session for participants should be incorporated into the survey flow. Adequate measures should be taken to safeguard tablets and a stable internet connexion should be guaranteed.

\section{P3.319 MALIGNANCIES SURVEILLANCE IN A HISPANIC HIV/AIDS COHORT BEFORE AND AFTER HAART}

doi:10.1136/sextrans-2013-051184.0772

'A M Mayor, 'D M Fernandez, 'E Rios-Oliveras, ${ }^{2} \mathrm{D}$ Zavala, 'R F Hunter-Mellado. ${ }^{1}$ Retrovirus Research Center, Universidad Central del Caribe, Bayamon, Puerto Rico, 2Puerto Rico Central Cancer Registry, San Juna, Puerto Rico

Background This study describes trends in malignancies as it relates to the availability of highly active antiretroviral therapy (HAART) in a Hispanic HIV/AIDS infected cohort.

Methods The database of 4,232 HIV infected adults followed in the Retrovirus Research Center at Bayamon, Puerto Rico was 
matched with the Puerto Rico Central Cancer Registry (PRCCR) database. AIDS and non-AIDS related malignancies standardised incidence rate (SIR) and 95\% CI in three time periods, defined as: 1992-1995 (pre-HAART), 1996-2002 (early-HAART), and 20032009 (late-HAART) were established. SIR evaluates a measure of risk related to the general population, and is defined as the ratio of observed to expected number of cancers. Expected counts were estimated by applying gender, age, and calendar years PRCCR's specific cancer incidence rates to our cohort.

Results Of the 296 malignancies found; $29.3 \%$ were women, $39.3 \%$ were injecting drug users and $42.9 \%$ were AIDS related cancers. The SIR for all malignancies in the pre-HAART period (10.15) decreased to 5.35 in the early-HAART, and to 2.04 in the late-HAART period. AIDS related malignancies SIRs decreased after HAART from 91.99 to 16.48; however, Kaposi's sarcoma (KS) and invasive cervical carcinoma (ICC) SIRs remained significantly higher in the late-HAART period (50.52 and 9.17). NonAIDS related malignancies' SIRs of the oral cavity/pharynx, liver, anus, vaginal, testis, Hodgkin's lymphomas (HL) and non-HL $(\mathrm{NHL})$ were significantly higher (SIRs > 3.30) in the late-HAART period.

Conclusion Availability of HAART in this Hispanic HIV/AIDS cohort has significantly decreased the malignancies risk. However, the higher incidence of KS, ICC and non-AIDS related malignancies in the late-HAART is suggestive of the role of additional oncogenic factors including sexual transmitted and injecting drug use infections. Aggressive intervention in the form of vaccines, risky practise reduction, early screening intervention and education needs to be incremented in this vulnerable population. Granted by 8G12MD007583, 8U54MD007587 and NPCR-CDC

\section{P3.320 UNLINKED ANONYMOUS TESTING FOR MONITORING HIV PREVALENCE IN SENTINEL GROUPS IN SLOVENIA, 2002-2011}

doi:10.1136/sextrans-2013-051184.0773

II Klavs, 'Z Kastelic, 'T Kustec, ${ }^{2} \mathrm{M}$ Poljak. ${ }^{1}$ National Institute of Public Health, Ljubljana, Slovenia; ${ }^{2}$ nnstitute of Microbiology \& Imunology, Medical Faculty, University of Ljubljana, Ljubljana, Slovenia

Background In addition to universal mandatory HIV/AIDS case reporting for HIV surveillance purposes, we also monitor HIV prevalence in several sentinel populations at higher risk (injecting drug users (IDU), men who have sex with men (MSM), patients with sexually transmitted infections (STI)) and one low risk group (pregnant women)

Methods During 2002-2011, we continuously sampled residual sera from STI patients tested for syphilis and every second year, residual sera from pregnant women screened for syphilis in several laboratories. Saliva specimens were continuously voluntarily obtained from IDU entering a substitution treatment programme and also, but for three months per year only, from IDU attending a harm reduction programme. Once per year, we obtained saliva specimens from MSM attending an MSM event. Specimens were labelled only with the type of sentinel population, sampling year, sex, and age group and were tested for antiHIV antibodies.

Results 1,066 saliva specimens were collected from MSM, 2,048 saliva specimens from IDU, 6,327 serum specimens from patients with STI, and 39,818 serum specimens from pregnant women. Annual prevalence estimates for MSM varied between $0 \%$ in 2002 and $7.6 \%$ in 2011 , for patients with STI between $0.2 \%$ in 2003 and $2.7 \%$ in 2008, and for pregnancies from $0 \%$ in 2003 and 2007 to $0.03 \%$ in 2011. Among IDU specimens, only two tested anti-HIV positive, one in 2010 and one in 2011 (0.4\% and $0.5 \%$ respectively).
Conclusions Our results indicate a disproportionally high and rising HIV infection prevalence among MSM that has increased above $5 \%$ in 2011. HIV infection prevalence among pregnancies has remained rather low, however, the highest ever $(0.5 \%)$ has been estimated in 2011. Promotion of safer sexual behaviour and HIV testing among MSM as well as positive prevention among MSM with HIV diagnosis are urgently needed. The introduction of HIV screening of pregnancies should be considered.

\section{P3.321 ACCEPTABILITY OF DATA COLLECTION ON MOBILE PHONES USING ODK SOFTWARE FOR SELF-ADMINISTERED SEXUAL BEHAVIOUR OUESTIONNAIRES}

doi:10.1136/sextrans-2013-051184.0774

'2Z A Kaufman, ${ }^{3} \mathrm{R}$ Hershow, ${ }^{3} \mathrm{~J}$ DeCelles, ${ }^{4} \mathrm{~K}$ Bhauti, ${ }^{1} \mathrm{~S}$ Dringus, ${ }^{2} \mathrm{~S}$ Delany-Moretlwe, 'D A Ross. 'London School of Hygiene and Tropical Medicine, London, UK; ${ }^{2}$ Wits Reproductive Health and HIV Institute, Johannesburg, South Africa; ${ }^{3}$ Grassroot Soccer, Cape Town, South Africa; ${ }^{4}$ Grassroot Soccer, Bulawayo, Zimbabwe

Background Previous studies in Africa and Latin America have shown that conducting surveys with mobile devices saves time and money compared to traditional pen-and-paper surveys. Open Data Kit $(\mathrm{ODK})$ is an open-source application suite for building, collecting, and managing data using Android-enabled phones or tablets. ODK enables complex skip patterns, multi-language implementation, multimedia, collection of GPS coordinates, and secure webbased data storage.

Methods Two self-administered sexual behaviour surveys were conducted on mobile phones using ODK: one with adolescents in Cape Town and Port Elizabeth, South Africa $(n=4485$, median age 15 years, 146-item questionnaire); one with adult male soccer players in Bulawayo, Zimbabwe ( $n=663$, median age 24 years, 71 -item questionnaire). Ten focus group discussions (FGDs) were conducted with participants and survey teams to assess acceptability. Additionally, participants were asked survey questions related to their comfort, understanding and satisfaction with this method of questionnaire administration. Non-response rates are reported for selected sensitive questions asked on both questionnaires.

Results FGDs found that participants and facilitators were comfortable and engaged when using the mobile phones. There was a strong feeling that using the mobile phone provided increased privacy and confidentiality when answering sensitive questions, compared to self-administered paper-based sexual behaviour surveys. In all, 4015 (78.1\%) participants reported preferring the mobile-phonebased survey to a pen-and-paper survey, while 716 (13.9\%) reported preferring pen-and-paper. Low non-response was observed in both studies for reported HIV testing (SA: $2.7 \%$; Zim: $1.8 \%$ ), condom use ever (SA: 8.7\%; Zim: 2.0\%), and previous STI experience (SA: $8.1 \%$; Zim: $2.6 \%$ ).

Conclusions Data capture on mobile phones using ODK had high acceptability among both South African adolescents and Zimbabwean men. Researchers conducting sexual behaviour surveys should consider data collection on mobile phones using ODK software as a potential data capture method.

\section{P3.322 LACK OF STANDARDISED REPORTING AND DOCUMENTATION OF MEASURES BEYOND ACCURACY IMPAIR OUALITY OF RESEARCH: EVIDENCE FROM SYPHILIS AND HIV POINT-OF-CARE DIAGNOSTICS}

doi:10.1136/sextrans-2013-051184.0775

T Chiavegatti, Y Jafari, N Pant Pai. McGill University and Health Center, Montreal, OC, Canada

Background HIV and Syphilis collectively infect about 70 million individuals. Both infections have long asymptomatic periods, 